\title{
Gain sideband splitting in dispersion oscillating fibers
}

\author{
Christophe Finot ${ }^{\mathrm{a}, *}$, Fang Feng ${ }^{\mathrm{a}}$, Yanne Chembo ${ }^{\mathrm{b}}$, Stefan Wabnitz ${ }^{\mathrm{c}}$ \\ ${ }^{a}$ Laboratoire Interdisciplinaire Carnot de Bourgogne, UMR 6303 CNRS-Université de Bourgogne, 9 avenue Alain Savary, BP 47870,21078 Dijon Cedex, France \\ ${ }^{\mathrm{b}}$ FEMTO-ST/Optics department, UMR 6174 CNRS-University of Franche-Comté, 16 route de Gray, 25030 Besançon Cedex, France \\ ${ }^{\mathrm{c}}$ Dipartimento di Ingegneria dell'Informazione, Università degli Studi di Brescia, via Branze 38, 25123 Brescia, Italy
}

\section{A R T I C L E I N F O}

\section{Article history:}

Received 21 April 2014

Revised 8 June 2014

Available online 10 July 2014

\section{Keywords:}

Dispersion oscillating fiber

Modulation instability

Four-wave mixing

\begin{abstract}
A B S T R A C T
We analyze the modulation instability spectrum in a varying dispersion optical fiber as a function of the dispersion oscillation amplitude. For large dispersion oscillations, we predict a novel sideband splitting into different sub-sidebands. The emergence of the new sidebands is observed whenever the classical perturbation analysis for parametric resonances predicts vanishing sideband amplitudes. The numerical results are in good quantitative agreement with Floquet or Bloch stability analysis of four-wave mixing in the periodic dispersion fiber. We have also shown that linear gain or loss may have a dramatic influence in reshaping the new sidebands.
\end{abstract}

(c) 2014 Elsevier Inc. All rights reserved.

\section{Introduction}

Modulation instability (MI) is a nonlinear process that has been widely investigated in various fields of physics including plasma, hydrodynamics and optics, to cite a few. In the presence of a high power continuous wave (CW), MI leads to the emergence and amplification of gain sidebands in the wave spectrum. In nonlinear fiber optics, such a process has been demonstrated in fibers with anomalous, constant group velocity dispersion (GVD) [1], as well as in normal GVD fibers by enabling the fulfillment of the nonlinear phase-matching condition through either fourth order dispersion [2], birefringence or a multimodal structure [3,4]. More recently, a renewed experimental and theoretical interest in MI studies has been stimulated by the availability of fibers presenting a longitudinal and periodic modulation of their dispersion properties [5]. Indeed, thanks to the periodic dispersion landscape, which leads to quasi-phase-matching (QPM) of the nonlinear four-wave mixing (FWM) process, MI sidebands can be observed even in the regime of normal average GVD of a dispersion-oscillating optical fiber (DOF) [6-8]. Recent experimental works have confirmed the QPM-induced MI process in the normal GVD regime of microstructured DOF around $1 \mu \mathrm{m}$ [5], as well as of non-microstructured highly nonlinear DOF at telecom wavelengths $[9,10]$.

To date, the role of the amplitude of the GVD oscillations on the MI spectrum of a DOF remains largely unexplored. In this work, we present a systematic study of the dependence of various sidebands which emerge at the output of a DOF, as the amplitude of the

\footnotetext{
* Corresponding author.

E-mail address: christophe.finot@u-bourgogne.fr (C. Finot).
}

dispersion variations grows progressively larger. We unveil the emergence of new sidebands, as well as their unexpected splitting in sub-sidebands. As we shall see, the emergence of new sidebands may be qualitatively described by extending to a continuum set of frequencies the analytical theory that was established in [11] for a discrete set of sidebands. We also present a detailed study of the influence of optical losses on the profile and splitting of MI sidebands in a DOF.

\section{Model and situation under investigation}

The evolution of the optical field $\psi$ in an DOF can be described by the nonlinear Schrödinger equation (NLSE) that includes both the Kerr nonlinearity $\gamma$ and a periodically varying second-order dispersion $\beta_{2}(z)$

$i \frac{\partial \psi}{\partial z}-\frac{\beta_{2}(z)}{2} \frac{\partial^{2} \psi}{\partial t^{2}}+\gamma|\psi|^{2} \psi+i \frac{\alpha}{2} \psi=0$

In the last section of this paper, we will also discuss the influence of linear losses that are included through the coefficient $\alpha$ (negative and positive values of $\alpha$ leading to distributed amplification and losses respectively). In Eq. (1) we did not include higherorder dispersion terms or Raman scattering. Nevertheless, we checked that these effects do not have a noticeable influence on the MI spectral dynamics that we are going to describe.

$\mathrm{MI}$ induced by the longitudinal variations of chromatic dispersion has been theoretically investigated before in a wide range of configurations, ranging from sinusoidal profiles with a spatial period of a few tens of meters [5,9], up to dispersion-managed systems with periods of several kilometers [6,12-14]. We consider 
in this contribution the specific case of DOF whose parameters are inspired by the previous numerical works which were carried out in the context of the transmission of high-speed telecommunication signals in dense dispersion managed links [15]. The fabrication of such a DOF is fully consistent with existing drawing techniques [16]. More precisely, the stepwise dispersion profile over one spatial period $\Lambda$ of the fiber under study is provided by the map

$$
\begin{cases}\beta_{2}(z)=\beta_{2 a v}+\beta_{2 a m p s} & \text { if } z<\Lambda / 2 \\ \beta_{2}(z)=\beta_{2 a v}-\beta_{2 a m p s} & \text { if } z>\Lambda / 2\end{cases}
$$

where $\beta_{2 a v}$ is the average dispersion of the fiber, and $\beta_{2 a m p s}$ is half of the peak-to-peak amplitude of the dispersion variation (the second order dispersion $\beta_{2}$ being related to the dispersion parameter $D$ by $D=-2 \pi c \beta_{2} / \lambda^{2}, \lambda$ being the wavelength and $c$ the light velocity). Using a Fourier decomposition, it is possible to decompose the profile (2) into a series of sinus functions having spatial frequencies multiple to $1 / \Lambda$. For the sake of simplicity, and since analytical solutions exist for this case, we concentrate here on the fundamental Fourier component, so that we may reduce the stepwise dispersion profile (2) to the following sinusoidal variation

$\beta_{2}(z)=\beta_{2 a v}+\beta_{2 a m p} \sin (2 \pi z / \Lambda)$

where $\beta_{2 a m p}$ is the corresponding amplitude of the dispersion variation: $\beta_{2 a m p}=4 \beta_{2 a m p s} / \pi$.

Let us consider here a DOF with a Kerr nonlinearity of $\gamma=2 \mathrm{~W}^{-1} \mathrm{~km}^{-1}$, a spatial period of the dispersion oscillation $\Lambda=1 \mathrm{~km}$, and a dispersion average value of $D_{a v}=-0.5 \mathrm{ps} / \mathrm{km} / \mathrm{nm}$ at the wavelength $\lambda_{0}=1550 \mathrm{~nm}$. The fiber is pumped by a CW with the average power $P=0.75 \mathrm{~W}$ at $\lambda_{0}$. The NLSE (1) is numerically solved by the standard split-step Fourier algorithm including a weak input white noise seed, and the results are averaged over 24 shots.

In the presence of sinusoidal longitudinal GVD variations, QPM of FWM or MI leads to the appearance of resonant gain sidebands, whose angular frequency shift relative to the pump can be analytically predicted, by assuming an indefinitely long fiber, as follows [6]:

$\Omega_{p}= \pm \sqrt{\frac{2 \pi p / \Lambda-2 \gamma P}{\beta_{2 a v}}}$

with $p=1,2,3 \ldots$. More recently, it has been shown that the gain experienced by the $p$ th sidebands after a propagation length $L$ can be predicted by the formula [11]:

$G_{p}^{d B}=10 \log _{10}\left\{\exp \left[2 \gamma P L\left|J_{p}\left(\frac{\beta_{2 a m p} \Omega_{p}^{2}}{2 \pi / \Lambda}\right)\right|\right]\right\}$

where $J_{p}$ is the Bessel function of order $p$. Note that Eqs. (4) and (5) are derived from Eqs. (1)-(3) in a perturbation limit, namely whenever $\left|\beta_{2 a m p}\right| \beta_{2 a v} \mid \ll 1[7,17]$. Therefore it is particularly interesting to study the domain of their validity in situations where the amplitude of the dispersion oscillations is equal or even much larger than the average dispersion. Note that indeed dispersion managed transmission systems almost always operate in the so-called strong management regime, namely the condition $\left|\beta_{2 a m p}\right| \beta_{2 a v} \mid \gg 1$ holds, which is precisely the opposite of the domain of validity of the perturbation theory that permits to derive Eqs. (4), (5).

\section{Influence of the amplitude of the dispersion fluctuation}

We start our study by investigating the influence of the amplitude of dispersion fluctuations $\beta_{2 a m p}$ (or the corresponding $D_{a m p}$ ) on the MI spectrum which is recorded after 12 spatial periods, i.e., a propagation distance of $12 \mathrm{~km}$. Results are plotted in Fig. 1 for three levels of the amplitude of GVD oscillations, namely

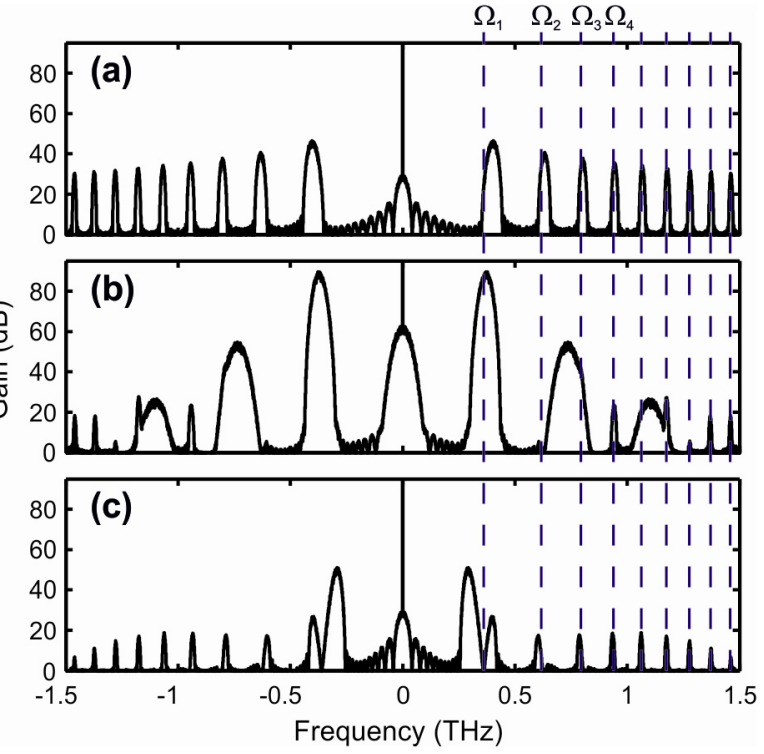

Fig. 1. Evolution of the output spectra recorded after $12 \mathrm{~km}$ of propagation for different values of the amplitude of the dispersion fluctuation: $D_{a m p}=0.5,1.7$ and $3.7 \mathrm{ps} / \mathrm{km} / \mathrm{nm}$, subplots (a), (b) and (c) and respectively. The vertical dashed lines represent the predictions from Eq. (4).

$D_{a m p}=0.5,1.7$ and $3.7 \mathrm{ps} / \mathrm{km} / \mathrm{nm}$, see subplots (a), (b) and (c), respectively. Our motivation in varying the amplitude of the dispersion oscillations rather than other parameters (such as the average dispersion or the longitudinal period, for example) is that a modification of the dispersion amplitude $\beta_{2 a m p}$ should in principle have no influence, on the basis of Eq. (4), on the position of the MI sidebands.

Indeed, for a relatively low level of dispersion fluctuations, that is $D_{a m p}=0.5 \mathrm{ps} / \mathrm{km} / \mathrm{nm}$ (case (a) of Fig. 1 ), we observe the generation of unequally spaced and narrow spectral sidebands, whose position is in qualitative agreement with the analytical predictions of Eq. (4) (see dashed vertical lines). The possibility of observing QPM-induced MI in such a situation, where a large set of spectral lines is generated, was experimentally confirmed in the work of Droques et al. [5].

However, for an increasing level of dispersion amplitude oscillations (e.g., $D_{a m p}=1.7 \mathrm{ps} / \mathrm{km} / \mathrm{nm}$, case (b) of Fig. 1), we observe two main features that lead to a very different structure of the output MI spectrum. We first notice from Fig. 1(b) that some of the spectral lines (for example, the lines corresponding to $p=2$ or $p=5$ ) have disappeared from the MI spectrum. Moreover, we may also point out in Fig. 1(b) the development of a new set of regularly spaced sidebands with a broader bandwidth. The first feature is explained by the evolution of the gain coefficient according to Eq. (5) which leads, for some values of the argument, to the annihilation of the sideband amplitude, as it was already confirmed experimentally in [11]. On the other hand, the second feature is linked to FWM between the pump wave and the first QPM sideband, and the subsequent cascading of the FWM process, as it was previously discussed and demonstrated experimentally in [9].

For an even higher amplitude of the dispersion oscillations, i.e. $D_{a m p}=3.7 \mathrm{ps} / \mathrm{km} / \mathrm{nm}$ (case (c) in Fig. 1), we observe instead of a single gain sideband, the unexpected emergence of a pair of sidebands around the angular frequency $\Omega_{1}$. The emergence of the new pair of sidebands is not explained by nonlinear mixing between the various other sidebands, but it is an intrinsic property of the MI gain spectrum of Eqs. (1) and (3) in the strong dispersion management regime.

To verify this statement, we may write the perturbed CW solution of the NLSE $(1,3)$ as $\psi(z, t)=[\sqrt{P}+u(z, t)] \exp \{i P z\}$, where we 
suppose $|u|^{2} \ll P$, so that we obtain the linearized equation for $u(Z, T)$

$i u_{Z}=\beta^{2} \frac{d(Z)}{2} u_{T T}+\left(u+u^{*}\right)$.

Here we defined $\beta^{2}=L_{n l} / L_{d}$, where the nonlinear length $L_{n l}=1 /(\gamma P)$, the dispersion length $L_{d}=t_{0}^{2} /\left|\beta_{2 a v}\right|$, and $t_{0}=1 p s$ is a reference time unit, so that $Z=z / L_{n l}$ and $T=t / t_{0}$. Moreover, $d(Z)=\left[1+\Delta \sin \left(2 \pi Z / \Lambda^{\prime}\right)\right]$ with $\Delta=\beta_{2 a m p} /\left|\beta_{2 a v}\right|$, and $\Lambda^{\prime}=\Lambda / L_{n l}$. By writing now the solution of (6) as the sum of Stokes and anti-Stokes sidebands, $u(Z, T)=a(Z) \exp \{i \Omega T)+b^{*}(Z) \exp \{-i \Omega T)$, one obtains the two coupled linear ordinary differential equations (ODEs) with periodic coefficients for $a(z)$ and $b(z)$

$a_{Z}=i \frac{\beta^{2}}{2} \Omega^{2} d(Z) a+i(a+b)$,

$b_{Z}=-i \frac{\beta^{2}}{2} \Omega^{2} d(Z) b-i(a+b)$.

These equations are equivalent to a single, second-order ODE or generalized Mathieu equation for the Stokes sideband $a(z)$ [7]. In the general case, the resulting periodic ODE cannot be solved analytically for an arbitrary strength of the dispersion oscillation: the parametric resonance conditions (4) being strictly valid only in the limit of small oscillations. However, the corresponding linear stability analysis can still be rigorously carried out numerically by the Floquet theory, which is analogous to Bloch wave theory in solid state physics $[18,19]$.

By defining the solution vector of ( 7$)$ as $\mathbf{s}=(a, b)$, and choosing the two fundamental initial conditions $\mathbf{s}_{1}(Z=0)=(1,0)$ and $\mathbf{s}_{2}(Z=0)=(0,1)$, one readily obtains from the solution of $(7)$ at $Z=\Lambda^{\prime}$ the principal solution matrix $S=\left[\mathbf{s}_{1}\left(\Lambda^{\prime}\right)^{\mathrm{t}}, \mathbf{s}_{2}\left(\Lambda^{\prime}\right)^{\mathrm{t}}\right]$ (where $t$ denotes the vector or matrix transpose). According to Floquet's theorem, the eigenvalues of $\mathrm{S}$, or Floquet's multipliers $\lambda=\exp \left(\eta_{F}+\right.$ $i \sigma)$, such that $|\lambda|>1$ yield the linear instability of the CW with respect to the growth of sidebands with frequency detuning $\Omega$. Since the scattering matrix after an integer number $\mathrm{n}$ of periods is simply $S^{n}$, one may relate the Floquet's multipliers to the usual MI gain $G$ after one period of the dispersion oscillation by the relation $G=2 \eta_{\mathrm{F}} / \Lambda^{\prime}$. Fig. 2 compares the MI gain spectra computed
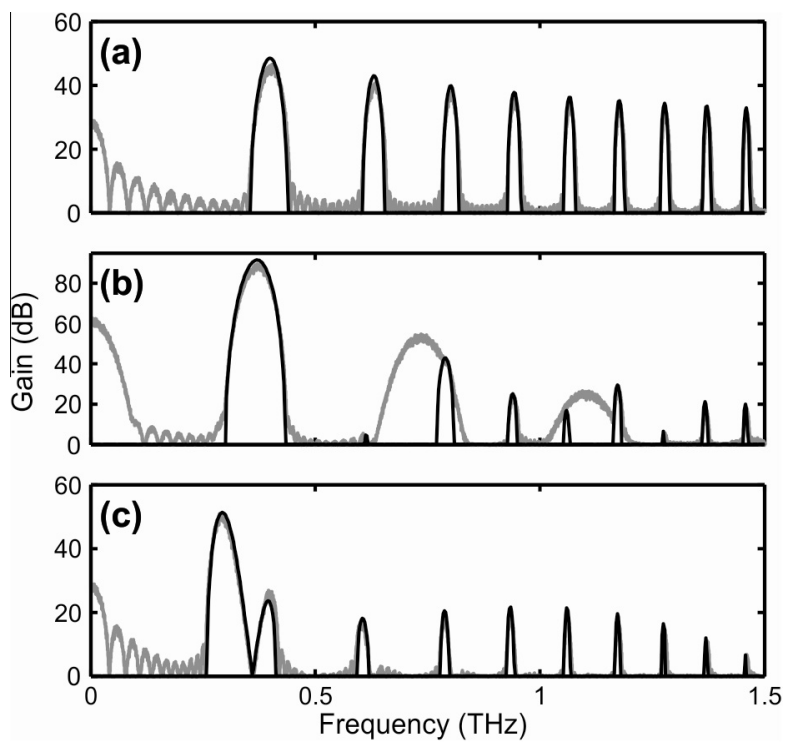

Fig. 2. Anti-Stokes sideband spectrum as Fig. 1. The results from the Floquet linear stability analysis of the NLSE with periodic dispersion (black lines) are compared with the results of the numerical integration of Eq. (1) (grey lines). Subplots (a), (b) and (c) are obtained for the same dispersion amplitude values of corresponding subplots in Fig. 1. from the Floquet's stability theory of Eq. (7), with the results of the numerical solutions of Eq. (1) as reported in Fig. 1, showing the MI gain after $n=12$ periods of the dispersion oscillation. As can be seen, there is an excellent agreement between the numerics and the Floquet's stability analysis for all values of the dispersion oscillation amplitudes. Clearly, in Fig. 2 the peaks resulting from multiple FWM cascading of the MI sidebands with the pump in Fig. 1(b) are absent from the MI spectrum computed with Floquet's theory, as cascading effects are not included in the linearized model of Eqs. (6 and 7).

In order to get a better overview of the role of the dispersion oscillation amplitude on the MI spectrum, we carried a more systematic study, which is summarized in Fig. 3(a). From this figure, several different behaviors can be clearly observed. Up to $D_{a m p}=1$ $\mathrm{ps} / \mathrm{km} / \mathrm{nm}$, the dynamics is similar to the case of Fig. 1(a). For $D_{a m p}$ between 1 and $2.75 \mathrm{ps} / \mathrm{km} / \mathrm{nm}$, FWM plays an important role, and it may lead to multiple cascading sidebands. In the range of dispersion oscillation amplitudes $D_{a m p}$ between 3.6 and $4.4 \mathrm{ps} / \mathrm{km} / \mathrm{nm}$, the splitting of two sidebands around the spectral position of the first QPM sideband can be observed (case of Fig. 1(c)). Such a behavior is also observed for $D_{a m p}$ above $6.5 \mathrm{ps} / \mathrm{km} / \mathrm{nm}$, whereas a case-(a) behavior is again restored for $D_{a m p}$ between 4.7 and $7 \mathrm{ps} / \mathrm{km} / \mathrm{nm}$.

The results of Fig. 3(a) are compared with those in Fig. 3(b), which are obtained from a similar dispersion map, involving a stepwise longitudinal dispersion modulation (Eq. (2)). We can see that qualitatively similar trends are observed in both Fig. 3(a) and (b), when taking into account the coefficient that is linked to the Fourier decomposition of a square function. We however note in Fig. 3(b) a decrease of the MI gain for relatively large values of $D_{a m p}$

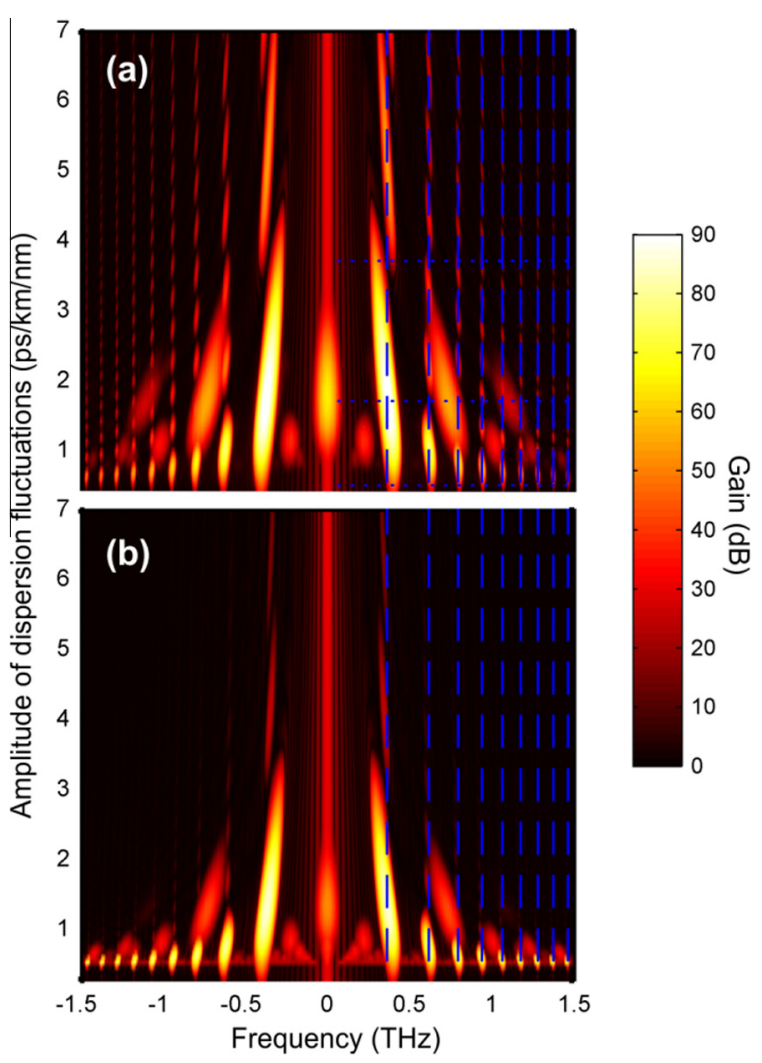

Fig. 3. Evolution of the output MI spectrum after a distance of $12 \mathrm{~km}$ according to the level of dispersion fluctuations. (a) Results obtained for a sinusoidal dispersion modulation. (b) Results obtained for a square dispersion modulation. The position of the sidebands predicted from Eq. (4) is plotted with vertical dashed lines. The spectra corresponding to cases of Fig. 1 are highlighted by dashed horizontal lines. 


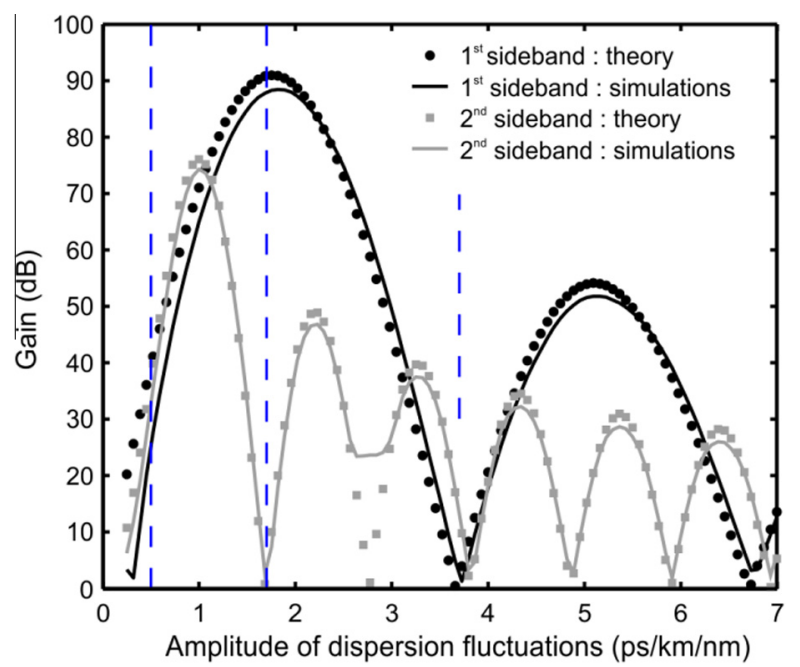

Fig. 4. Evolution with $D_{a m p}$ of gain at the frequencies predicted by Eq. (4) for the first and second QPM sidebands (black and grey colors respectively). Results obtained from numerical simulations (solid lines) are compared with analytical predictions from Eq. (5) (circles and squares). Positions of the three cases reported in Fig. 1 are marked with dashed vertical lines.

From Fig. 3, we may observe a non-monotonic evolution of the gain as a function of the dispersion oscillation amplitude. Thus we have studied in more details the evolution of the gain at the discrete set of QPM wavelengths which are predicted by Eq. (4). The corresponding results are summarized in Fig 4, where we compare the analytical predictions of Eq. (5) with the gain that is evaluated from numerical simulations at $\Omega_{1}$ and $\Omega_{2}$. Several points can here be stressed. First, Fig. 4 provides the confirmation of the explanation for the behavior which is observed in the case of Fig. 1(b): for $D_{\text {amp }}=1.7 \mathrm{ps} / \mathrm{km} / \mathrm{nm}$, the gain of the second sideband vanishes. Whereas the gain of the first sideband reaches its maximum value, thus stimulating cascaded FWM between the pump and this sideband.

From Fig. 3(a), we may also notice that for $D_{a m p}$ around $3 \mathrm{ps} / \mathrm{km} /$ $\mathrm{nm}$, the gain induced by the FWM between the 1st QPM band and the pump overlaps with the second QPM sideband, thus explaining the discrepancy between numerics and analytical theory (4) for the position of the second sideband as it is observed in Fig. 3. Next, we may observe from Fig. 4 that, for the case reported in Fig. 1(c), both numerics and analytic formula (5) predict that the gain at the first sideband position $\Omega_{1}$ vanishes. An important conclusion that we can draw from Fig. 4 is that the agreement between the numerical simulations and the analytical predictions (4-5) is excellent over the whole range of dispersion oscillation amplitudes under study. Therefore, we may conclude that the observation in the case of Fig. 1(c) of two sidebands on either side of the angular frequency $\Omega_{1}$ is not in contrast with the perturbation theory analytical results of Eqs. (4) and (5).

From Figs. 3(a) and 4, we may note that a similar situation also appears for dispersion oscillations with an amplitude around $D_{a m p}=6.7 \mathrm{ps} / \mathrm{km} / \mathrm{nm}$, where again the theory predicts a vanishing gain for the first sideband: the emergence of two new idebands is also observed in this case.

In order to better understand the emergence of the two neighboring sidebands as observed in Fig. 1(c), we have plotted in Fig. 5 a magnification of the MI spectrum around the first QPM sideband as obtained from numerical simulations: see Fig. 5(a), that we compare with Fig 5(b), which reports the following analytical expression

$$
G^{d B}(\omega)=10 \log _{10}\left(\exp \left[2 \gamma P L\left|J_{1}\left(\frac{\beta_{2 a m p} \omega^{2}}{2 \pi / \Lambda}\right)\right|\right]\right)
$$

The above formula is an extension of Eq. (5), which was initially aimed at describing the gain exclusively at the discrete set of the QPM frequencies $\Omega_{p}$, to a continuum range of frequencies $\Omega$ in the vicinity of a QPM frequency. Despite the use of Eq. (8) is not fully rigorous (as this formula was derived for $\omega=\Omega_{1}$ only), we can see from Fig. 5 that it provides interesting insights in the existence of the two sidebands on either side of the angular frequency $\Omega_{1}$.

Indeed, as it is shown in panel 6(a), when comparing the structure of the gain around $\Omega_{1}$, we may point out that the analytical expression (8) reproduces well the inner slope of the two sidebands. The validity of the analytical approximation (8) is also confirmed by carrying out a similar analysis for a different value of the amplitude of $D_{a m p}=6.7 \mathrm{ps} / \mathrm{km} / \mathrm{nm}$, such that sideband splitting is also observed, see Fig. 6(b). A better quantitative agreement with the numerics can in each case be achieved by the Floquet linear stability analysis (see Figs. 5(c) and 6).

From the previous analysis of the case reported in Fig. 1(c), we may conclude that the existence of two new sub-sidebands on
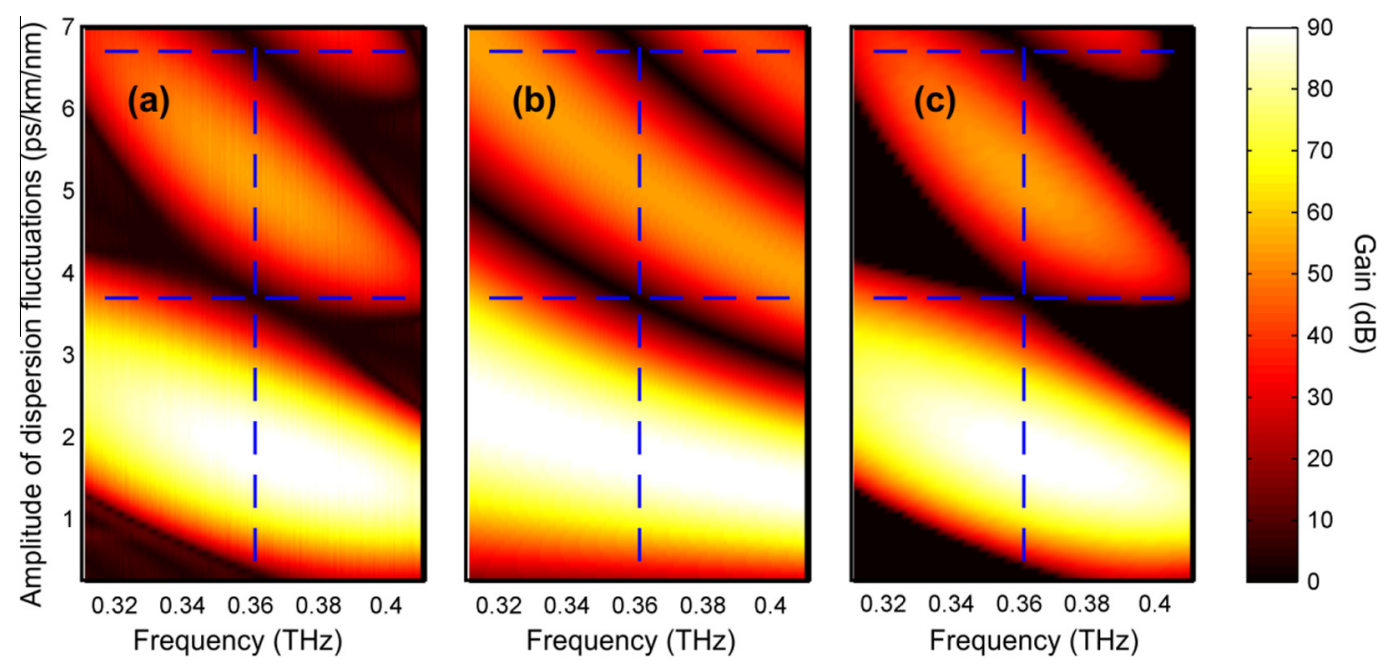

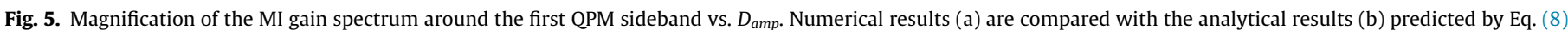

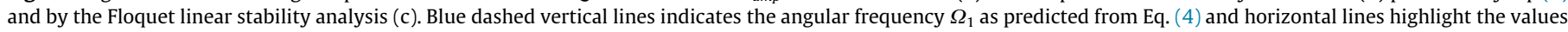
of dispersion fluctuations that are then used in Fig. 6. 


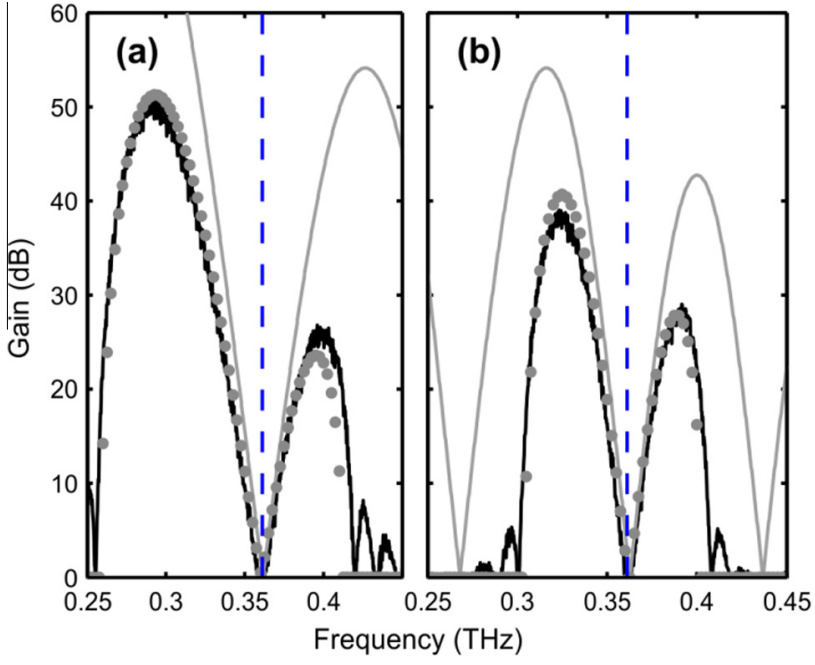

Fig. 6. Output spectrum after $12 \mathrm{~km}$ of propagation obtained from numerical simulations (black line), from analytical equation (grey line) and from the Floquet linear stability analysis (grey circles): (a) $D_{a m p}=3.7 \mathrm{ps} / \mathrm{km} / \mathrm{nm}$; (b) $D_{a m p}=6.7 \mathrm{ps}$ / $\mathrm{km} / \mathrm{nm}$.

either side of the angular frequency $\Omega_{1}$ does not violate the prediction of both Eqs. (4) and (5), which forecast zero gain at $\Omega_{1}$ in this case. The emergence of these sidebands is thus directly linked to their splitting from the first QPM sideband, whose gain at $\Omega_{1}$ is precisely zero. Quite interestingly, the gain of the two sidebands in the vicinity of $\Omega_{1}$ can be approximated by simply extending the validity of Eq. (5) to a continuum range of frequencies.

\section{Influence of optical losses/gain}

In the second part of this article, we shall examine the influence of fiber linear gain or losses on the resulting output MI spectrum after $12 \mathrm{~km}$ of propagation. For simplicity, we limit our analysis for the significant case where sideband splitting is observed, i.e., $D_{\text {amp }}=3.7 \mathrm{ps} / \mathrm{km} / \mathrm{nm}$.

The impact of gain can be seen in Fig. 7(a): here we observe, for a linear gain of $0.05 \mathrm{~dB}$ per $\mathrm{km}$, the amplification of the inner
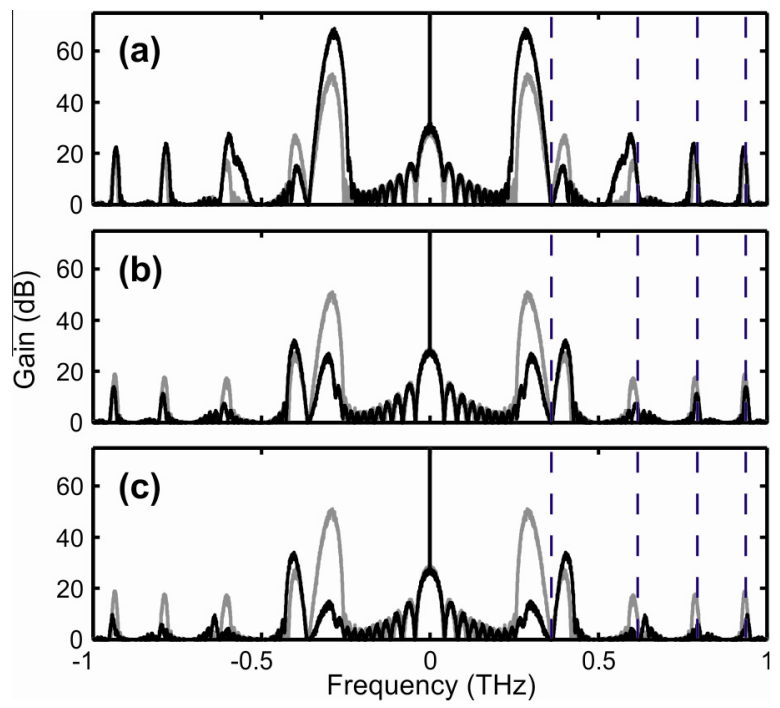

Fig. 7. Evolution of the output spectra after $12 \mathrm{~km}$ of propagation for different levels of losses or gain (solid black lines): (a) $0.05 \mathrm{~dB} / \mathrm{km}$ of gain. (b) $0.05 \mathrm{~dB} / \mathrm{km}$ of loss. (c) $0.1 \mathrm{~dB} / \mathrm{km}$ of loss. Results obtained without losses are plotted with grey solid lines. sub-sideband (i.e. the sideband located at frequencies below $\Omega_{1}$ ), whereas quite surprisingly the MI gain of the outer sub-sideband decreases (i.e. the sideband located at frequencies above $\Omega_{1}$ ). We also observe in Fig. 7(a) the emergence of a new FWM-induced sideband, located close to the second QPM sideband.

On the other hand when dealing with the impact of linear losses, we may notice that even with a level of losses as low as $0.05 \mathrm{~dB}$ per km (Fig 7(b)), the MI gain of the inner sideband experiences a dramatic decrease. To the contrary, the outer sideband undergoes a slight gain increase. For a further increase of the level of losses (i.e., $0.1 \mathrm{~dB} / \mathrm{km}$, Fig. 7(c)), the gain of the inner sideband drops down to a few $\mathrm{dB}$ only, whereas the gain of the outer sub-sideband does not change significantly. Let us point out that, contrary to several other studies which were earlier carried out with reference to dispersion managed transmission systems [14], we do not consider here a periodic amplification of the field.

A more systematic study on the impact of linear loss on the MI spectrum is summarized on Fig. 8(a): these results confirm that losses have a very different impact on inner and outer subsidebands. To further highlight the radically different evolution of the gain for the two sidebands as a function of the linear gain/ loss coefficient, we have reported the respective behavior in Fig. 8(b). As it can be seen, the inner sideband is enhanced by the presence of linear gain, but it is extremely sensitive to an even low level of losses. To the contrary, the outer sub-sideband suffers from the presence of linear gain; whereas and its amplitude is increased by the presence of a moderate amount of losses. For the usual levels of fiber losses $(>0.2 \mathrm{~dB} / \mathrm{km})$, the inner sidebands cannot be clearly distinguished, so that only the outer subsidebands will be observed in practice.
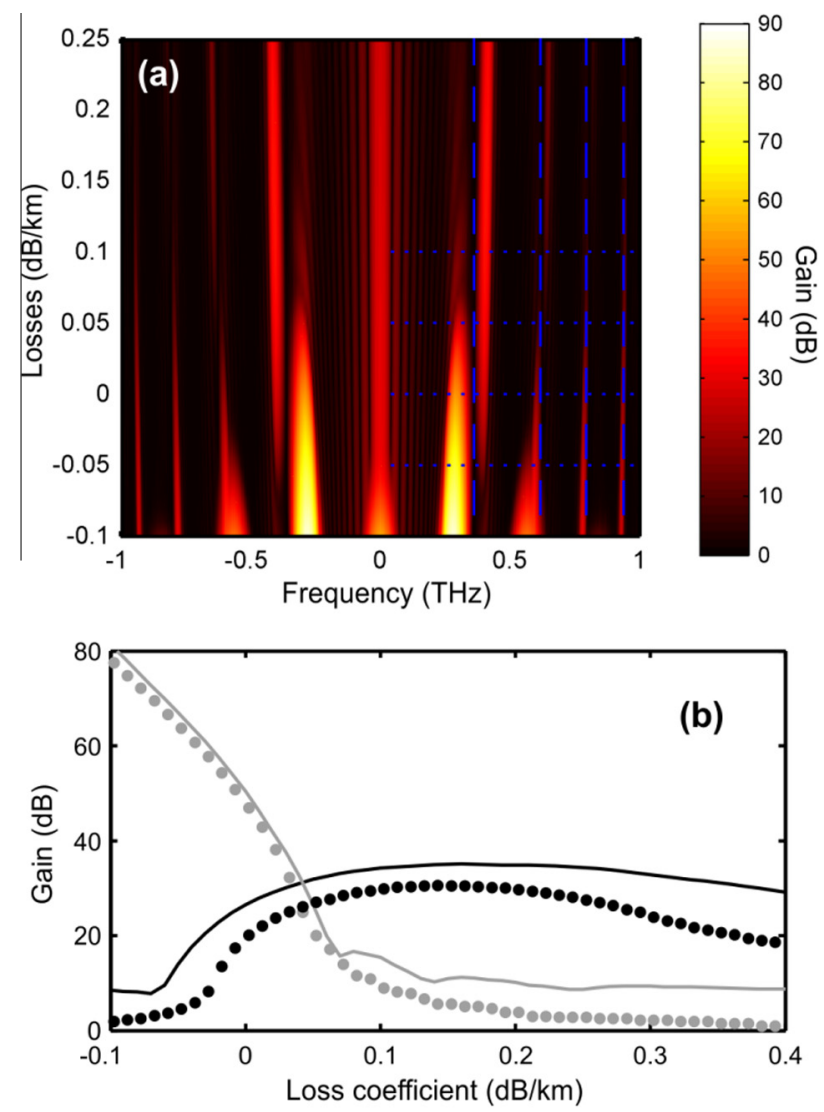

Fig. 8. (a) Evolution of the output spectrum after $12 \mathrm{~km}$ of propagation according to the level of linear losses/gain. (b) Evolution of the parametric gain experienced by inner and outer sidebands (grey and black lines or symbols, respectively) according to the level of linear losses/gain. The numerical results (solid lines) are compared with results based on Eq. (9) (dots). 
The different dependence on gain and loss of the two sub-sidebands may be qualitatively explained by the fact that the angular frequency of the resonant sidebands predicted by Eq. (4) is power dependent, higher powers favoring lower values of $\Omega_{1}$. A more complete picture of the influence of the pump level on the detailed evolution of the first sideband is provided on Fig. 9(a), where the Floquet-based linear stability analysis (carried out without loss) is used to compute the power dependent gain spectra $G_{F}^{d B}(\omega, P)$. As it can be seen, the first sideband splits in two spectrally shifted sub-sidebands in the vicinity of the point of zero gain at the frequency predicted by Eq. (4). In other words, Fig. 9(a) reveals a striking development of inner and outer branches out of a given sideband of the MI gain spectrum. The progressive increase of the $\mathrm{CW}$ power with propagation distance, which occurs in the presence of distributed amplification, tends to progressively shift $\Omega_{1}$ towards the inner band, which is thus enhanced. To the contrary, linear losses lead to a progressive shift of $\Omega_{1}$ towards higher values, thus favoring the development of the outer sub-sideband. Note that such a behavior is just the opposite to what can be observed in the standard MI process (i.e., for a fiber with constant dispersion).

In order to get slightly more quantitative insights on the impact of loss or gain, we clearly cannot rely on Eq. (8) as the overall shape
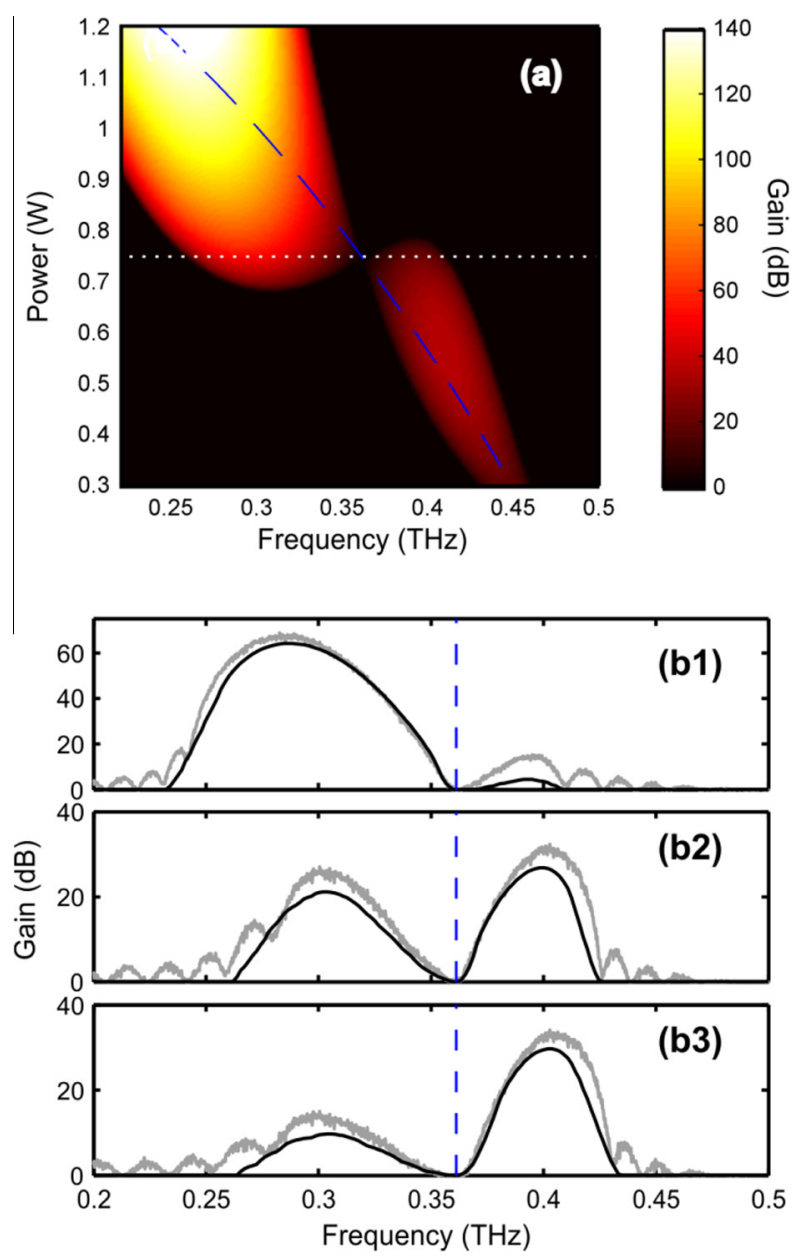

Fig. 9. (a) Evolution of the first sideband after $12 \mathrm{~km}$ of propagation according to the input power of the $\mathrm{CW}$ and as predicted by the of Floquet linear stability analysis without any loss or gain. The dashed blue line represents the central frequency of the sideband as predicted by Eq. (4), whereas the dotted horizontal line stands for the operating power that has been considered in previous section. (b) Details of the first sideband for the same levels of loss as Fig. 7: results from numerical integration of Eq. (1) (grey lines) are compared with results from Eq. (9) (black line). provided by this expression is not power dependent. Therefore, to evaluate roughly the influence of $\alpha$ on the MI spectrum, we used the following approximate approach: we consider the output gain spectra (in $\mathrm{dB}$ ) after $n$ periods of the dispersion oscillation as the sum of the previously computed gain spectra experienced over a single spatial period for a power at the input of the period that increases/decreases exponentially with propagation length:

$G^{d B}(\omega, \alpha)=\sum_{i=1 . . n} G_{F}^{d B}\left(\omega, P_{0} e^{-\alpha \Lambda} i\right)$

Resulting spectra for values of $\alpha$ similar to Fig. 7 are plotted in Fig. 9(b). A pretty good agreement with the numerical integration of Eq. (2) is observed, which confirms our qualitative explanation. A satisfactory agreement is also reached in Fig. 8(b), so that we may conclude that Eq. (9) reproduces well the trends which are obtained from the full and rigorous simulation of the NLSE (1).

It also proves interesting to study the longitudinal evolution of the MI gain of the two sub-sidebands in the presence of either linear gain or loss. The corresponding results are summarized in Fig. 10, and confirm that the previous conclusions remain valid at other values of the propagation distance. We note however here a limit of the approach based on Eq. (9): being based on a sum of positive terms, the gain predicted by Eq. (9) can intrinsically only increase upon propagation. Therefore, this is not possible to reproduce with Eq. (9) the short scale fluctuations of the gain. Moreover, instead of the non-monotonic evolution of gain with distance recorded for the inner sideband for a loss of $0.1 \mathrm{~dB} / \mathrm{km}$ (see Fig. 10(a1)), with the approach provided by Eq. (9) we observe a plateau (see Fig. 10(b1)).
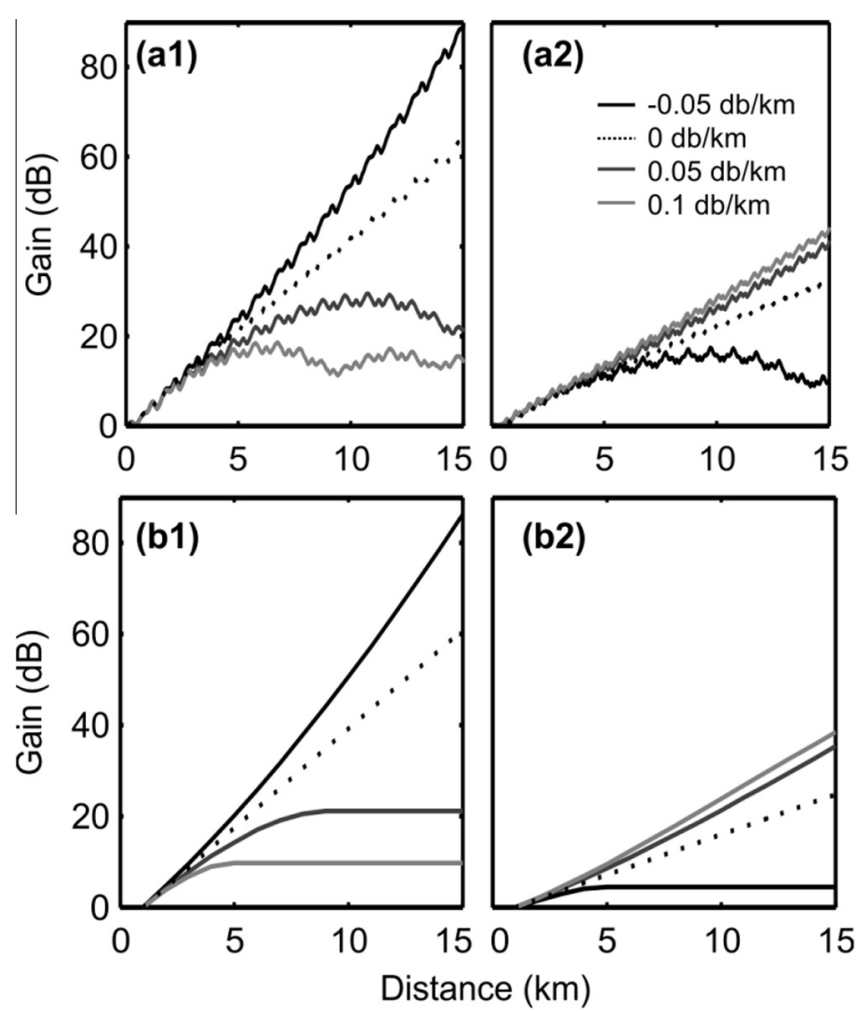

Fig. 10. Longitudinal evolution of the gain for the inner and outer sub-sidebands (panels (a1,b1) and (a2,b2) respectively) for different levels of attenuations/losses: $-0.05,0,0.05$ and $0.1 \mathrm{~dB} / \mathrm{km}$, solid black, dotted black, dark grey and light grey lines respectively. Results from numerical simulations $(\mathrm{a} 1, \mathrm{a} 2)$ are compared with the results predicted by Eq. (9) (b1, b2). 


\section{Discussion and conclusion}

In this work we have studied the evolution of the modulation instability spectrum in an optical fiber with periodically varying dispersion, as a function of the dispersion oscillation amplitude. Existing theory for DOFs predicts, in addition with the well-known modulation instability that is observed in fibers with constant dispersion, the emergence of a discrete, infinite set of parametric resonance sidebands both in the normal and in the anomalous dispersion regime. However these parametric resonance sidebands are obtained in a perturbative limit of relatively small dispersion oscillations. Hence there is in principle no guarantee about the survival and existence of parametric resonances in DOFs with relatively large amplitude dispersion oscillations, or in the so-called strong dispersion management regime.

As a matter of fact, we found that even in the limit of dispersion oscillation with an amplitude much larger than the average fiber dispersion, the predictions of the perturbative analysis generally hold surprisingly well. Nevertheless, in situations where the standard perturbative analysis predicts that the parametric sidebands have a vanishing amplitude, we observed an unexpected emergence of new, higher order sidebands which results from the splitting of the corresponding original parametric sideband. Our numerical results are in good quantitative agreement with a Floquet stability analysis of four-wave mixing in a DOF. Furthermore, we have also shown that linear gain or loss may have a dramatic influence in reshaping the relative amplitude of each of the new sidebands.

From the application point of view, the observed sideband splitting may be considered as generating a double-sideband, suppressed carrier modulation format (where by carrier we mean the original first order parametric resonant sideband). Moreover, analogous phenomena are expected to occur in other types of quasi-phase-matched nonlinear interactions, for example in periodically poled quadratic materials $[20,21]$. From a fundamental viewpoint, the present sideband splitting appears to be a universal feature of strongly parametrically driven nonlinear dynamical systems. Indeed, in non-autonomous dynamical systems (e.g., a parametrically forced pendulum), as the amplitude of the periodic forcing grows larger, instability regions open up in the parameter space which are known as Floquet's or resonance tongues. Such instability domains can be described by means of the Mathieu or the Hill's equations: in this context, MI sideband splitting is analogous to the bifurcations of the tongues which lead to the so-called pockets of instability [22,23].

\section{Acknowledgments}

We thank Julien Fatome, Stéphane Pitois, Kamal Hammani and Alexej Sysoliatin for stimulating discussions. We acknowledge the financial support of the Conseil Regional de Bourgogne (Pari Photcom), the funding of the Labex ACTION program (ANR-11-
LABX-01-01), and the Italian Ministry of University and Research (grant no. 2012BFNWZ2).

\section{References}

[1] K. Tai, A. Hasegawa, A. Tomita, Observation of modulational instability in optical fibers, Phys. Rev. Lett. 56 (1986) 135-138.

[2] S. Pitois, G. Millot, Experimental observation of a new modulational instability spectral window induced by fourth-order dispersion in a normally dispersive single-mode optical fiber, Opt. Commun. 226 (2003) 415-422.

[3] E. Seve, P. Tchofo-Dinda, G. Millot, M. Remoissenet, J.M. Bilbault, M. Haelterman, Modulational instability and critical regime in a highly birefringent fiber, Phys. Rev. A 54 (1996) 3519-3533.

[4] G. Millot, S. Pitois, P. Tchofo-Dinda, M. Haelterman, Observation of modulational instability induced by velocity-matched cross-phase modulation in a normally dispersive bimodal fiber, Opt. Lett. 22 (1997) 1686-1688.

[5] M. Droques, A. Kudlinski, G. Bouwmans, G. Martinelli, A. Mussot, Experimental demonstration of modulation instability in an optical fiber with a periodic dispersion landscape, Opt. Lett. 37 (2012) 4832-4834.

[6] N.J. Smith, N.J. Doran, Modulational instabilities in fibers with periodic dispersion management, Opt. Lett. 21 (1996) 570.

[7] F.K. Abdullaev, S.A. Darmanyan, A. Kobyakov, F. Lederer, Modulational instability in optical fibers with variable dispersion, Phys. Lett. A 220 (1996) $213-218$.

[8] A. Armaroli, F. Biancalana, Tunable modulational instability sidebands via parametric resonance in periodically tapered optical fibers, Opt. Express 20 (2012) 25096-25110

[9] C. Finot, J. Fatome, A. Sysoliatin, A. Kosolapov, S. Wabnitz, Competing fourwave mixing processes in dispersion oscillating telecom fiber, Opt. Lett. 38 (2013) 5361-5364.

[10] F. Feng, J. Fatome, A. Sysoliatin, Y.K. Chembo, S. Wabnitz, C. Finot, Wavelength conversion and temporal compression of pulse train using dispersion oscillating fibre, Electron. Lett. 50 (2014) 768-770.

[11] M. Droques, A. Kudlinski, G. Bouwmans, G. Martinelli, A. Mussot, Dynamics of the modulation instability spectrum in optical fibers with oscillating dispersion, Phys. Rev. A 87 (2013) 013813.

[12] F. Consolandi, C. De Angelis, A.-D. Capobianco, G. Nalesso, A. Tonello, Parametric gain in fiber systems with periodic dispersion management, Opt. Commun. 208 (2002) 309-320.

[13] A. Kumar, A. Labruyere, P. Tchofo Dinda, Modulational instability in fiber systems with periodic loss compensation and dispersion management, Opt. Commun. 219 (2003) 221-232.

[14] S. Ambomo, C.M. Ngabireng, P.T. Dinda, A. Labruyère, K. Porsezian, B. Kalithasan, Critical behavior with dramatic enhancement of modulational instability gain in fiber systems with periodic variation dispersion, J. Opt. Soc. Am. B 25 (2008) 425-433.

[15] J. Fatome, C. Fortier, S. Pitois, Practical design rules for single-channel ultra high-speed dense dispersion management telecommunication systems, Opt. Commun. 282 (2009) 1427-1434.

[16] L. Provost, C. Moreau, G. Mélin, X. Rejeaunier, L. Gasca, P. Sillard, P. Sansonetti, Dispersion-Managed Fiber with low chromatic dspersion slope, Optical Fiber Communication Conference (OFC), 2003, pp. 151-153.

[17] L.D. Landau, W.M. Lifshitz, Mechanics, Pergamon, Oxford, 1973.

[18] E.A. Coddington, N. Levison, Theory of Ordinary Differential Equations, McGraw-Hill, New York, 1955.

[19] S. Trillo, S. Wabnitz, Bloch wave theory of modulational polarization instabilities in birefringent optical fibers, Phys. Rev. E 56 (1997) 1048-1058.

[20] J.F. Corney, O. Bang, Plane waves in periodic, quadratically nonlinear slab waveguides: stability and exact Fourier structure, J. Opt. Soc. Am. B 19 (2002) $812-821$.

[21] J.F. Corney, O. Bang, Complete modulational-instability gain spectrum of nonlinear quasi-phase-matching gratings, J. Opt. Soc. Am. B 21 (2004) 617621.

[22] H.W. Broer, M. Levi, Geometrical aspects of stability theory for Hill's equations. Arch. Rat. Mech. An. 131 (2004) 225-240.

[23] H.W. Broer, C. Simo, Resonance tongues in Hill's equations: a geometric approach, J. Diff. Eqns. 166 (2000) (2000) 290-327. 